\title{
Uveal lymphoid infiltrates: immunohistochemical evidence for a lymphoid neoplasia
}

\author{
DAVID BEN-EZRA, ' JOSE A SAHEL, "* NANCY L HARRIS, + I HEMO,' \\ AND DANIEL M ALBERT²
}

From the 'Department of Ophthalmology, Hadassah University Hospital, Jerusalem, Israel; 'David G Cogan Eye Pathology Laboratory, Massachusetts Eye and Ear Infirmary, Boston, USA: 'Clinique Ophtalmologique, Hospices Civils de Strasbourg, Strasbourg, France; and ${ }^{+}$Department of Pathology, Massachusetts General Hospital, Boston, USA

SUMMARY A 67-year-old man presented with a diffuse choroidal and ciliary body infiltrate, suggesting clinically and ultrasonographically a diffuse uveal melanoma. After enucleation both morphological and immunohistochemical data were highly suggestive of a diffuse, low-grade B cell lymphoma or lymphoplasmacytic immunocytoma. The diffficulties of clinical and histopathological differential diagnosis of uveal lymphoid infiltrates are emphasised. In view of the excellent life prognosis of these tumours, treatment of the patient should be directed towards the preservation of ocular function.

Until recently most cases of uveal lymphoproliferation have been diagnosed from histopathological examination. ${ }^{1-3}$ This condition, currently designated as 'reactive lymphoid hyperplasia,' was considered benign until recent immunological techniques raised the suspicion of a low-grade lymphomatous proliferation.

We report a clinicopathological study of a case of diffuse, unilateral, uveal lymphoproliferative disease in which both morphological and immunological patterns strongly suggested the diagnosis of small lymphocytic lymphoma with a plasmacytoid differentiation.

\section{Case report}

A 67-year-old man was seen on 5 October, 1986 complaining of seeing shadows in front of his right eye and changing refractive status for six months. Past medical history was negative.

Corrected visual acuity was 20/60 OD, 20/20 OS. In the right eye slit-lamp examination showed scleral thinning over a dark mass, $2 \mathrm{~mm}$ posterior to the nasal limbus. Transillumination disclosed the presence of a ring mass. The anterior segment and

Correspondence to Daniel M Albert. MD. Howe Laboratory of Ophthalmology, Massachusetts Eye and Ear Infirmary. 24.3 Charles Street. Boston. MA 02114. USA. vitreous appeared normal. Fundus examination revealed a ciliary and a subretinal mass extending to the neighbourhood of the temporal arcades, with a shallow subretinal detachment. Ultrasound disclosed a diffuse tumour largest at the ciliary body level. The mass showed low internal reflectivity, with a suspicion of intrascleral infiltration nasally. No choroidal excavation was seen (Fig. 1). No extraocular tumour was detected. CT scan of the brain, globes, and orbits showed a diffuse mass in the right globe (Fig. 2).

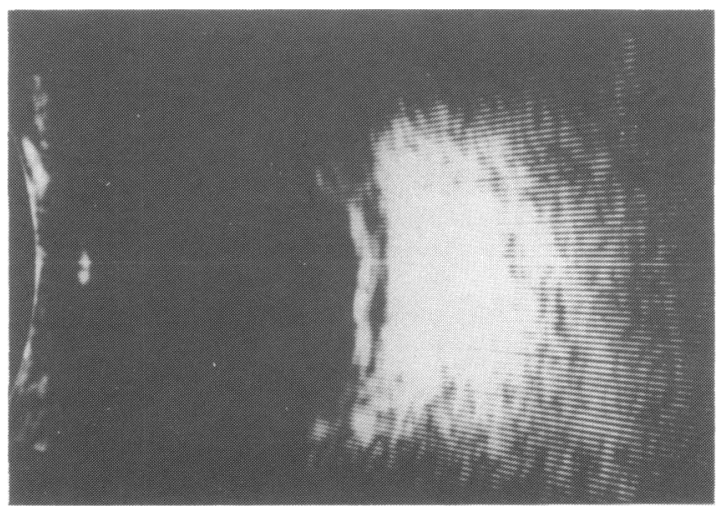

Fig. 1 Echogram B: diffuse tumour with low internal reflectivity. 


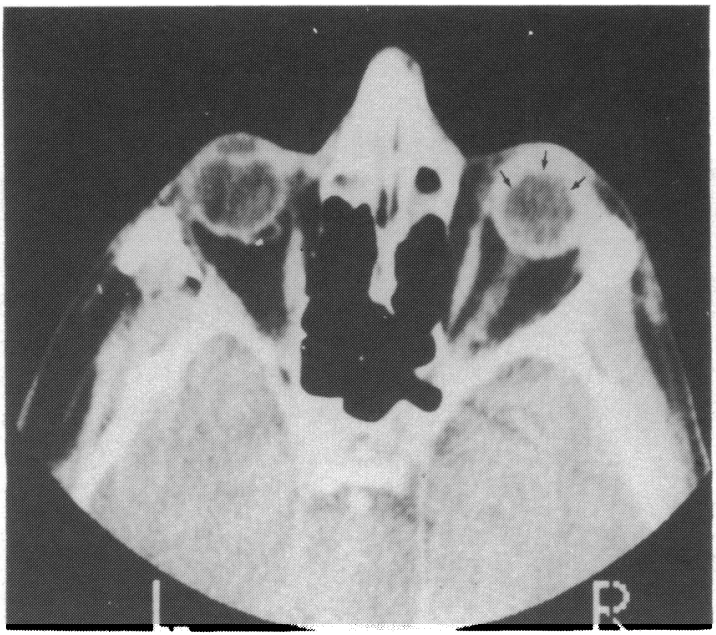

Fig. 2 CT scan: Diffuse involvement of the choroid and parts of orbit and optic nerve. Arrows indicate tumour.

Ancillary laboratory tests were negative, including spleen and liver scans, biochemical blood tests, blood cell counts, and protein electrophoresis. Quantitative immunoelectrophoresis showed only elevated IgM $4370 \mathrm{mg} / \mathrm{l}$ (normal 650-2800).

Three months later the suspected uveal melanoma progressed toward the posterior pole with retinal detachment and a drop of visual acuity in the right eye to 20/300. Enucleation was performed on 14 December, 1986.

In view of the histopathological findings a bone marrow aspirate was performed. This provided no evidence for a marrow involvement by lymphoma.

\section{HISTOPATHOLOGY}

The choroid and the ciliary body were extensively infiltrated by a lymphoid proliferation (Fig. 3). No extraocular extension was seen on serial sections. The infiltrate was focally follicular, with reactiveappearing follicles containing transformed lymphocytes, numerous mitoses, and phagocytic macrophages (Fig. 4). In the interfollicular regions there were diffusely infiltrating lymphocytes and plasma cells with no cytological abnormality (Fig. 5). A few plasmacytoid cells contained intranuclear periodic acid Schiff positive inclusions (Dutcher bodies) (Fig. 6).

The retina was partially detached, with an eosinophilic subretinal exudate. No optic nerve, retinal, or extraocular involvement was seen.

Immunoperoxidase staining with monoclonal antibodies to human immunoglobulin heavy and light chains were carried out on paraffin embedded sections with an avidin biotin peroxidase conjugate

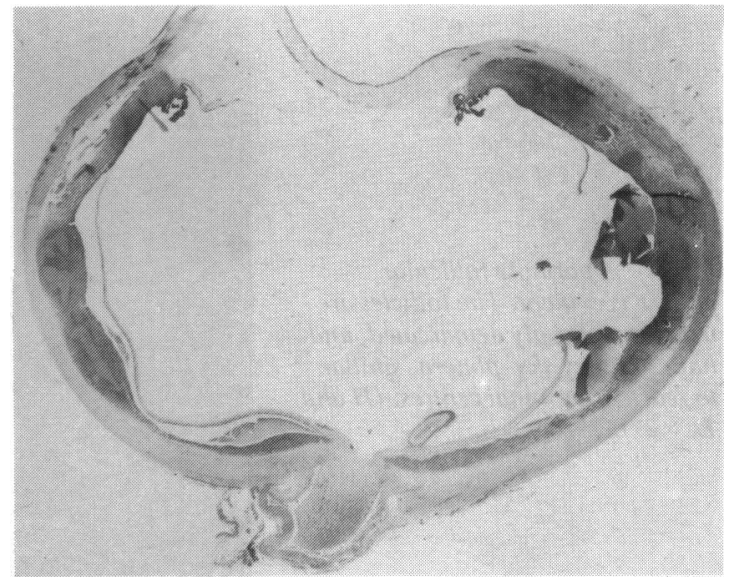

Fig. 3 Whole amount of eye showing diffuse involvement of choroid and ciliary body by a lymphoid proliferation. The retina is part detached. (H and $E, \times 1.5)$.

(Vector Laboratories, California). Plasma cells, plasmacytoid cells, and Dutcher bodies within and around the follicles showed a marked predominance of cytoplasmic IgM staining. Only scattered $\lambda$ and IgG positive cells were present (Fig. 7 A-C).

Transmission electron microscopy studies were performed from the paraffin blocks. In the germinal centres macrophages, plasma cells, and small lymphocytes were seen, a finding consistent with the light microscopic finding of polymorphism. In addition some plasmacytoid cells showed cytoplasmic disorganisation, characterised by eccentrically located cytoplasmic organelles like those found by Knowles and Jakobiec in their group IV lymphomas (Fig. 8).

The diagnosis of small lymphocytic lymphoma with plasmacytoid differentiation was made, or lymphoplasmacytic immunocytoma."

\section{Discussion}

Uveal lymphoid infiltrates (ULI) (so-called lymphoid hyperplasia) present difficulties in differential diagnosis from diffuse uveal melanoma. Except for two reports, ${ }^{25}$ in most cases of ULI the diagnosis was first established after enucleation or, in one instance, through a biopsy of the ciliary body. ${ }^{127-4}$ The age distributions of the patients and most symptoms are similar in ULI and diffuse uveal melanoma.' The diagnosis of lymphoid infiltrates should be suspected at an early stage if creamy choroidal infiltrates with indistinct edges, unassociated with retinal detachment or alteration of the retinal pigment epithelium are present. ${ }^{3}$ Other suggestive features include 
Fig. 4 A partially follicular pattern is evident. The follicles are irregular, sharply demarcated, and have a 'starry sky' pattern, similar to reactive germinal centres. ( $H$ and $E, \times 27)$.

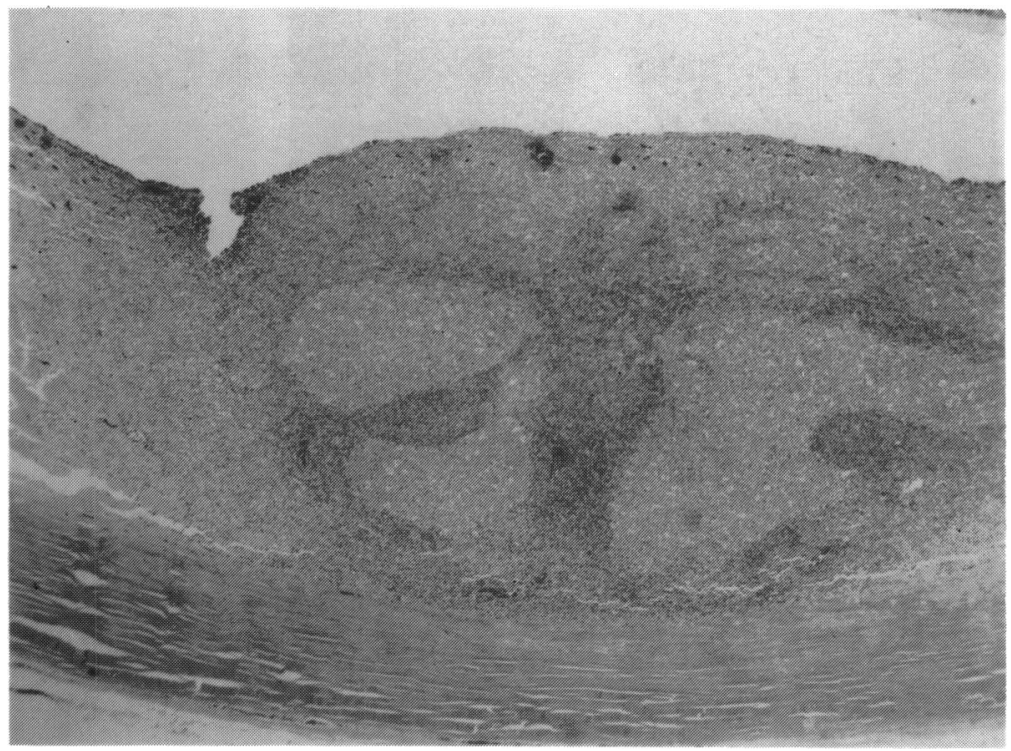

associated extraocular foci, glaucoma (66\%), inflammatory signs $(56 \%)$, or bilateral involvement $(10 \%))^{\prime}$ Desroches et $a l^{2}$ and Jakobiec et al ${ }^{\beta}$ emphasise the value of ultrasound findings: low internal reflectivity, lack of vascular pulsation, absence of choroidal excavations, integrity of the sclera. Jakobiec et al were able to reach the diagnosis through a biopsy of extraocular lesions. ${ }^{3}$ In our case, owing to the lack of clinical, ultrasonographic, and biological distinctive features, a differential diagnosis of reactive lymphoid hyperplasia seemed unlikely.

ULI present a problem in histological diagnosis. The first reported cases were identified as lymphosarcomas; Zimmermann reclassified them as reactive lymphoid processes. ${ }^{7}$ In 1972 Ryan et al reviewed 21 cases and described the classical histological pattern of what they called 'reactive lymphoid hyperplasia of the uvea.'

Fig. 5 Higher magnification of follicle and interfollicular region, showing reactive-appearing follicle. Interfollicular cells are predominantly small lymphocytes. ( $H$ and $E, \times 270$ ).

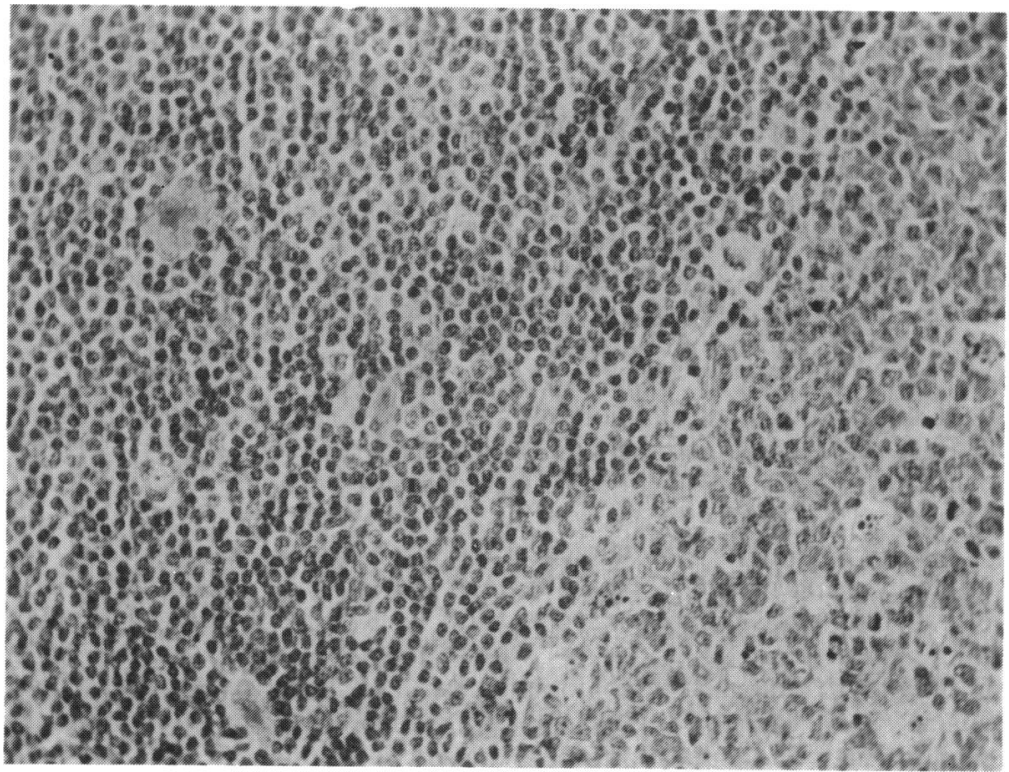




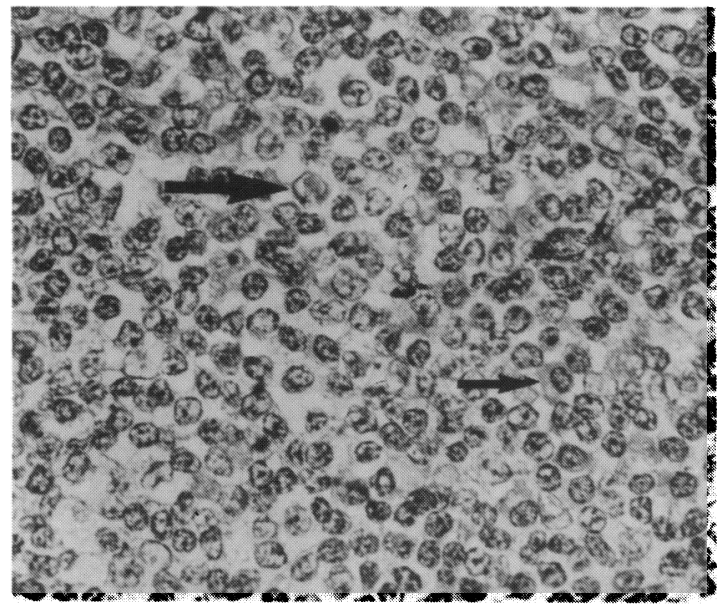

Fig. 6 The infiltrate contains a mixture of small lymphocytes and plasma cells (small arrow). Some cells have intranuclear PAS-positive inclusions (Dutcher bodies, large arrow). (PAS, ×450).

Yet the reservations held by all the authors on the purely morphological identification of extranodal lymphoproliferative diseases apply to the uvea as well. These doubts stem from (1) the lack of architectural features usually present in lymph nodes,

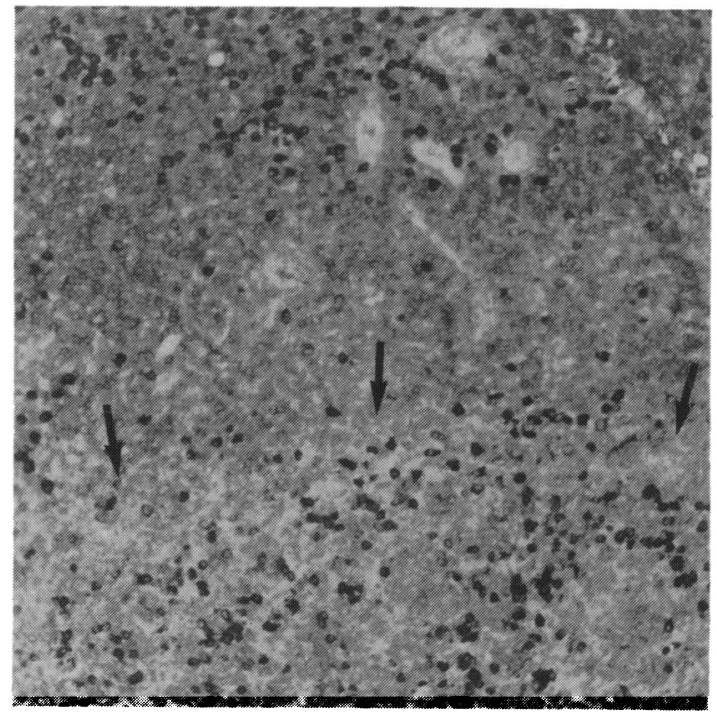

Fig. 7A Immunoperoxidase stains on paraffin-embedded sections. A $(\times 100)$ : Antibody to x light chain shows cytoplasmic staining of cells within the follicle centre (arrows), and in the intermacular region, particularly the choriocapillaries (top). B $(\times 100)$ : Antibody to $\lambda$ light chain (same field) shows only rare positive cells. $\mathrm{C}(\times 280)$.

Antibody to $\mu$ heavy chain is similar to $x$; occasional Dutcher bodies stain positively (arrow).

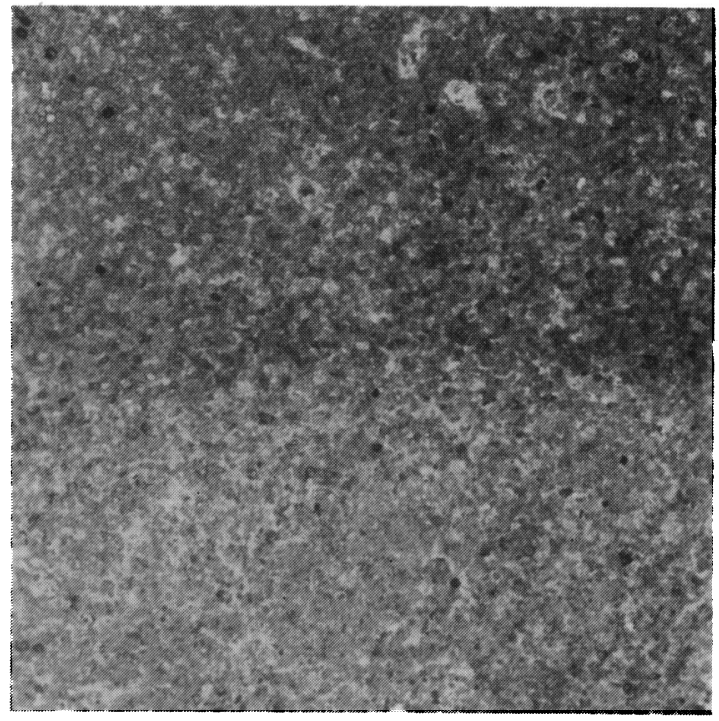

Fig. 7B

and (2) uncertainty as to the significance of germinal centres, Dutcher bodies, and plasma cells. ${ }^{101}$

The diagnosis of lymphoma in extranodal lymphoid infiltrates that lack cytological abnormalities is a major problem in pathology. ${ }^{10 / 4}$. Immunohistochemical studies have thrown light on this question. Reactive infiltrates contain a mixture of cells with both $x$ and $\lambda$ immunoglobulin light chains

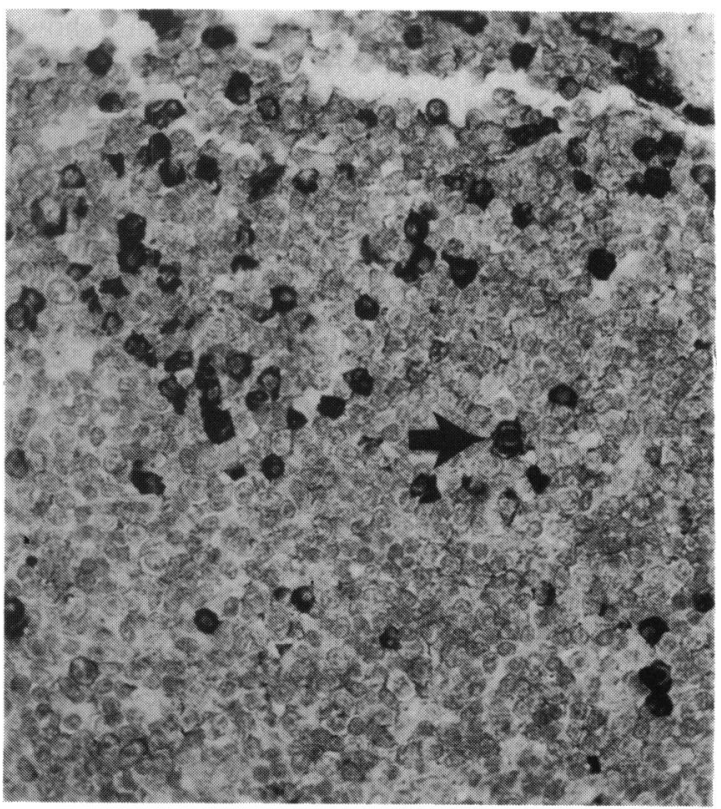

Fig. 7C 
Fig. 8 Transmission electron micrograph: plasma cell with eccentric organelles (see text). $(\times 11800)$.

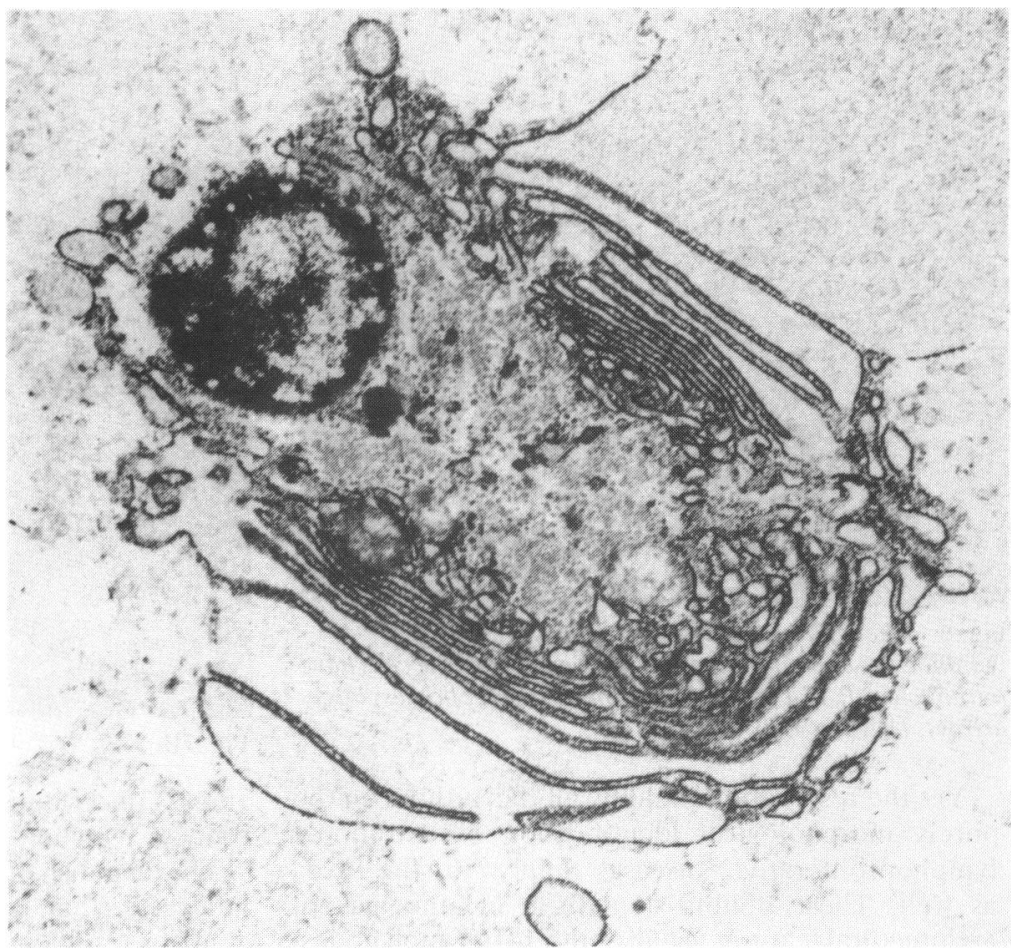

(polyclonal), while neoplasms consist of cells expressing only one light chain $-x$ or $\lambda$ (monoclonal). The application of this technique to extranodal lymphoid infiltrates, particularly in the orbit, has shown that most lesions previously diagnosed as pseudolymphoma are monoclonal B lymphocyte proliferations ${ }^{111111} 1.2-1.5$ - that is, low-grade $\mathrm{B}$ cell lymphomas.

These observations can now be applied to uveal lymphocytic infiltrates. Mauriello et $\mathrm{al}^{4}$ reported that in a series of cases of ULI studied by the immunoperoxidase technique the majority could be reclassified as well differentiated lymphoplasmocytic lymphomas. Histopathological examination of extraocular lesions in Jakobiec and colleagues' series provided morphological arguments for a lymphoid neoplasia. ${ }^{3}$ These tumours have a good prognosis for life, as has been reported for similar lesions occurring in the orbit. ${ }^{131416}$ Systemic involvement is rare. ${ }^{4}$

Consequently the accurate classification of these lesions as benign or malignant, which remains difficult, approaches an academic rather than practical consideration. The patients must be continually followed up for systemic disease. In its absence, the management of low-grade uveal lymphomas should be directed to the preservation of ocular function. Low-grade lymphomas of the conjunctiva and the ocular adnexa respond well to doses of radiation therapy that preserve visual function. ${ }^{17}$ High-grade lymphomas of the uveal tract have been successfully treated by radiotherapy. ${ }^{16} \mathrm{We}$ therefore advocate the use of corticosteroids as a diagnostic tool, ${ }^{2}$ followed by an associated low-dose irradiation if the diagnosis is very likely.

\section{References}

1 Ryan SJ, Zimmermann LE, King FM. Reactive lymphoid hyperplasia. An unusual form of intraocular pseudotumor. Ophthalmology 1972: 76: 652-71.

2 Desroches G, Abrams GW, Gass JDM. Reactive lymphoid hyperplasia of the uvea. A case with ultrasonographic and computed tomographic studies. Arch Ophthalmol 1983; 101: $725-8$.

3 Jakobiec FA, Sacks E, Kronish JW, Weiss T, Smith M. Multifocal static creamy choroidal infiltrates: an early sign of lymphoid neoplasia. Ophthalmology 1987; 94: 397-406.

4 Mauriello JA, Langloss JM, Weiner JM, Zimmermann LE. A clinicopathologic and immunohistologic study of lymphoproliferative lesions of the uveal tract. Invest Ophthalmol Vis Sci 1982: 22(ARVO suppl): 171.

5 Knowles DM. Jakobiec FA. Ocular adnexal lymphoid neoplasms: clinical, histopathologic, electron microscopic, and immunologic characteristics. Hum Pathol 1982; 13: 148-62.

6 The Non-Hodgkin's Lymphoma Pathologic Classification Project. National Cancer Institute sponsored study of classifications of non-Hodgkin's lymphomas: summary and description of a working formulation for clinical usage. Cancer 1982; 49: $2112-35$. 
7 Zimmermann LE. Lymphoid tumors. In: Boniuk M. ed. Ocular and adnexal tumors: new and controversial aspects. St Louis: Mosby, 1974: 438-46.

8 Crookes GP. Mullaney J. Lymphoid hyperplasia of the uveal tract simulating uveal melanoma. Am J Ophthalmol 1967: 63: 962-7.

9 Ryan SJ. Frank RN, Green WR. Bilateral inflammatory pseudotumours of the ciliary body. Am J Ophthalmol 1971: 72: 586-91.

10 Harris NL, Pilch BZ, Bhan AF, Harmon DC, Goodman ML. Immunohistologic diagnosis of orbital lymphoid infiltrates. Am J Surg Pathol 1984; 8: 83-91.

11 Evans HL. Extranodal small lymphocytic proliferation: a clinicopathologic and immunohistochemical study. Cancer 1982: 49: 84-96.

12 Jakobiec FA. The orbit. In: Spencer WH, ed. Ophthalmic pathology. Philadelphia: Saunders, 1986: 3: 2663-711.
13 Garner A, Rahi AHS, Wright JE. Lymphoproliferative disorders of the orbit: an immunological approach to diagnosis and pathogenesis. Br J Ophthalmol 1983; 87: 561-9.

14 Ellis JH. Banks PM. Camphell RJ, Liesegang TJ. Lymphoid tumors of the ocular adnexa: clinical correlation with the working formulation, classification and immunoperoxidase staining of paraffin sections. Ophthalmology 1985: 92: 1311-24.

15 Molenaar WM, Schwarze EW, Lennert K. An immunological study of germinal centres in four ophthalmic immunocytomas. Virchows Arch (A) 1983: 399: 141-8.

16 Corriveau C, Easterhook M. Payne D. Lymphoma simulating uveitis. Can J Ophthalmol 1986; 21 : 144-9.

17 Barthold HJ II, Harvey A. Markoc AM, Brady LW. Augsburger JJ. Shields JA. Treatment of orbital pseudotumors and lymphomas. Am J Clin Oncol 1986; 9: 527-32.

Accepted for publication 10 April 1989. 